\title{
Erratum: Electroweak-like baryogenesis with new chiral matter
}

\author{
Kohei Fujikura, ${ }^{a}$ Keisuke Harigaya, ${ }^{b}$ Yuichiro Nakai ${ }^{c}$ and Isaac R. Wang ${ }^{d}$ \\ ${ }^{a}$ Department of Physics, Tokyo Institute of Technology, \\ 2-12-1 Ookayama, Meguro-ku, Tokyo 152-8551, Japan \\ ${ }^{b}$ School of Natural Sciences, Institute for Advanced Study, \\ Princeton, New Jersey, 08540 U.S.A. \\ ${ }^{c}$ Tsung-Dao Lee Institute and School of Physics and Astronomy, \\ Shanghai Jiao Tong University, 800 Dongchuan Road, Shanghai, 200240 China \\ ${ }^{d}$ Department of Physics and Astronomy, Rutgers University, \\ Piscataway, New Jersey, 08854 U.S.A. \\ E-mail: fuji@th.phys.titech.ac.jp, keisukeharigaya@ias.edu, \\ ynakai0930@gmail.com, isaac.wang@rutgers.edu
}

ERRATUM TO: JHEP07(2021)224

ArXiv EPrint: 2103.05005

The name of the corresponding author of "Electroweak-like baryogenesis with new chiral matter" has changed from Ruoquan Wang to Isaac R. Wang.

His email address has changed from ruoquan.wang@rutgers.edu to isaac.wang@rutgers.edu.

Open Access. This article is distributed under the terms of the Creative Commons Attribution License (CC-BY 4.0), which permits any use, distribution and reproduction in any medium, provided the original author(s) and source are credited. 